\title{
Avaliação da eficácia do Programa Rede Mãe Paranaense
}

\author{
Assessment of effectiveness of the Rede Mãe Paranaense Program \\ Daiane Ribeiro dos Santos', Cláudia Silveira Viera', Ana Tereza Bittencourt Guimarães', Beatriz \\ Rosana Gonçalves de Oliveira Toso', Rosângela Aparecida Pimenta Ferrari²
}

DOI: $10.1590 / 0103-1104202012405$

RESUMO A avaliação da implantação de políticas e programas para melhorias na saúde infantil é essencial. Este estudo pretende avaliar a eficácia do Programa Rede Mãe Paranaense, criando um índice avaliativo para os objetivos do programa referentes ao seguimento da criança, a fim de estipular o grau em que foram concretizados. Foram entrevistadas 139 mulheres usuárias do programa. A pesquisa utilizou dados do instrumento elaborado e analisou a Caderneta de Saúde da Criança. A análise de dados foi realizada a partir da comparação entre frequências relativas obtidas no estudo e as propostas do programa, calculando-se a razão entre essas frequências para se obter o índice avaliativo, ou seja, para descobrir quanto foi alcançado da meta estipulada. As variáveis foram categorizadas em domínios definidos no programa, gerando um índice avaliativo para cada um deles. No domínio 'Satisfação Materna', a meta foi 68\% atingida; no 'Acompanhamento na Puericultura', foram atingidos 67\%; na 'Qualidade e Resolubilidade na Assistência', $55 \%$; e na 'Estratificação de Risco', somente $6 \%$ da meta foram alcançados. Conclui-se que os índices avaliativos retratam baixa eficácia no seguimento da criança. O estudo evidenciou a necessidade de revisão das estratégias utilizadas no seguimento da saúde da criança para melhorar os índices assistenciais na Atenção Primária à Saúde.

PALAVRAS-CHAVE Avaliação de programas e projetos de saúde. Saúde da criança. Indicadores básicos de saúde. Atenção Primária à Saúde.

ABSTRACT Assessing the implementation of policies and programs for improving child health is essential. This study aimed at assessing the effectiveness of the Rede Mãe Paranaense Program, creating an evaluative index for the objectives of the program regarding child follow-up, in order to stipulate the degree to which they were implemented. 139 women who used the program were interviewed. The research used data from the elaborated instrument and analyzed the Child Health Handbook. Data analysis was performed by comparing the relative frequencies obtained in the study and those proposed by the program, calculating the ratio between these frequencies to obtain the evaluative index, i.e., to find out how much was achieved from the stipulated goal. The variables were categorized into domains defined in the program, generating an evaluative index for each of these. In the 'Maternal Satisfaction' domain, the goal was 68\% achieved; in 'Follow-up in childcare', 67\% was achieved; in 'Quality and Resolvability in Care' 55\%; and 'Risk Stratification', only $6 \%$ of the goal was achieved. It is concluded that the evaluative indexes portray low efficacy in following the

1 Universidade Estadual do Oeste do Paraná (Unioeste) - Cascavel (PR), Brasil.

dainurse.enf@gmail.com

2 Universidade Estadual de Londrina (UEL) - Londrina (PR), Brasil. child. The study evidenced the need to review the strategies used in the follow-up of child health to improve care indices in Primary Health Care.

KEYWORDS Program evaluation. Child health. Health status indicators. Primary Health Care. 


\section{Introdução}

O sistema de saúde brasileiro, a partir do final dos anos 1980, se configurou como único e universal, denominando-se Sistema Único de Saúde (SUS). Esse sistema buscou superar os programas de saúde desenvolvidos até então, os quais tinham como característica a fragmentação da assistência, o que resultava em baixo impacto nos indicadores de saúde da população, especialmente da mulher e da criança'.

Não é recente a tentativa de mudanças no cenário de morbimortalidade infantil no Brasil. Para tanto, surgiram várias políticas ao longo das últimas décadas. Contudo, as políticas até então eram focadas em intensa medicalização do processo de nascimento, o que, apesar do conhecimento e desenvolvimento tecnológico envolvidos, ainda favorecia a elevada taxa de morbimortalidade materna, perinatal e infantil, que para alguns autores se constitui como 'um paradoxo perinatal brasileiro'2.

Com o processo de industrialização, na primeira metade do século $\mathrm{XX}$, a preocupação no País girava em torno do crescimento econômico, que contrastava com as altas taxas de mortalidade infantil. Os programas de saúde infantil eram direcionados a grupos com maior vulnerabilidade e estruturados de modo vertical, sem articulação com políticas sociais e distribuição de renda, e ainda desvalorizavam as necessidades específicas da população. Percebe-se, assim, que programas de saúde fragmentados e implantados de maneira impositiva pelo Estado resultam em políticas pouco resolutivas e desconexas da realidade ${ }^{\mathbf{1 , 3}}$.

Isso demonstrou a urgente necessidade de reorientação do modelo de atenção ao pré-natal, parto, nascimento, puerpério e seguimento da criança após o parto, embasado nas evidências de que ofertar maior número de procedimentos técnicose consultas não tem trazido resultados satisfatórios, e a qualidade do seguimento da criança ainda está comprometida. Uma das estratégias utilizadas em países desenvolvidos tem sido buscar a articulação dos programas de saúde com as políticas sociais, visto que a condição de saúde dos indivíduos está diretamente relacionada a condições sociais, que refletirão futuramente nos indicadores de morbimortalidade materno-infantil. Isto reforça a lógica de que as maiores taxas de mortalidade materno-infantil estão nos países subdesenvolvidos ${ }^{4}$.

Nesse sentido, pautadas nas necessidades locorregionais, nas taxas de mortalidade materno-infantil, bem como na tentativa de superar o modelo fragmentado de saúde vigente, emergem as Redes de Atenção à Saúde. Na saúde materno-infantil, surge o Rede Cegonha, pela Portaria ${ }^{\circ}$ $1.459 / 2011$, a partir da qual cada Estado passou a implementar o seu próprio programa.

No Paraná, em 2012, foi implantado o Programa Rede Mãe Paranaense (PRMP), que tem entre seus objetivos a redução da mortalidade materno-infantil e a garantia do funcionamento da rede para esse grupo, em todo o Estado. Observou-se redução na taxa de mortalidade infantil no Estado, a partir da implantação do PRMP, de 11,6/mil Nascidos Vivos (NV), em 2012, para 10,4/mil NV, em 2017. Porém, a maioria dos óbitos registrados foi considerada evitável - aproximadamente 66,6\%, de 2011 a 2013 , e $35,7 \%$, de 2014 a $2016^{5}$.

As estratégias utilizadas pelo PRMP para garantir uma rede de atenção materno-infantil com padrões de qualidade, organizada em todas as regiões com equidade e com mínima ocorrência de óbitos materno-infantis foram: melhorar a qualidade e a responsabilidade da assistência ao pré-natal, parto e puerpério; implantar e implementar a Linha Guia da Atenção MaternoInfantil; implantar a estratificação de risco em todos os níveis de atenção para a gestante e para a criança; vincular as gestantes aos hospitais de referência, conforme estratificação de risco; melhorar a assistência ao pré-natal de risco e o acompanhamento das crianças de risco menores de 1 ano; implementar transporte sanitário eletivo e de urgência para gestantes e crianças em situação de risco; disponibilizar exames de apoio e diagnóstico de pré-natal padronizados pela linha guia ${ }^{5}$.

Cada programa social e de saúde implementado tem suas metas e objetivos, que devem ser atingidos ao longo de sua implementação. Deste modo, torna-se imprescindível mensurar 
o alcance de tais programas em longo prazo, para que se obtenham subsídios capazes de impulsionar o desenvolvimento ou aprimoramento das políticas públicas.

Uma vez que o PRMP foi recentemente implantado, os estudos acerca do mesmo se referem a aspectos específicos do programa como consultas de pré-natal, gestão, seguimento da criança (cobertura vacinal), risco e mortalidade infantil, obtido por meio de dados nos Sistemas de Informação em Saúde (SIS) ${ }^{6,7}$, outros estudos investigaram as taxas de mortalidade infantil após implantação do PRMP,9. Ainda, estudos se debruçaram na análise do programa sob a ótica dos profissionais médicos ${ }^{10}$ e enfermeiros ${ }^{11}$, porém estes estudos não abordaram a visão do usuário, demonstrando assim uma lacuna neste aspecto.

Ainda, considerando o acompanhamento da criança após o nascimento, proposto pelo PRMP como estratégia que pode promover a saúde da criança e, consequentemente, reduzir os óbitos evitáveis, bem como a ausência de estudos sobre o PRMP relativos a este objetivo, é incontestável a necessidade de avaliar como está o desenvolvimento do seguimento da saúde da criança no programa.

Nesse contexto, é pertinente a avaliação do referido objetivo do PRMP quanto à sua eficácia, a qual busca analisar a "relação entre os objetivos e instrumentos explícitos de um programa e seus resultados efetivos"12(34). A avaliação das metas estipuladas pelo programa como meio de identificar sua eficácia pode ser analisada sob pontos de vista distintos, ou seja, pelo olhar dos proponentes do programa e gestores da atenção primária com foco no impacto econômico desde sua implantação, dos profissionais de saúde que avaliam o êxito do programa, a partir da exequibilidade de sua implantação e do alcance dos objetivos, ou pelos usuários beneficiados pelo programa.

Dessa forma, emerge a necessidade de identificar a eficácia do PRMP relativa ao seguimento da criança após seis meses de vida, por meio da avaliação do alcance de seus objetivos e metas, de acordo com a ótica materna. Assim, este estudo tem como objetivo avaliar a eficácia do PRMP, a partir da criação de um Índice
Avaliativo (IA) para cada objetivo do programa, referente ao seguimento da criança na Atenção Primária à Saúde, para que seja possível mensurar se os objetivos e metas foram concretizados desde sua implantação.

\section{Material e métodos}

O município em estudo tem população estimada em 319.608 habitantes, e está localizado na Região Oeste do Estado do Paraná, conforme dados do Instituto Brasileiro de Geografia e Estatística (IBGE), publicados em agosto de 2017. A Secretaria Municipal de Saúde (Sesau) possui Atenção Primária à Saúde do município com 12 Unidades Básicas de Saúde (UBS) e 31 Unidades de Saúde da Família (USF), que acompanham as crianças nas consultas de puericultura ${ }^{13}$.

O presente estudo apresentou abordagem quantitativa com desenho transversal. Para cálculo amostral, considerou o total de 2.288 partos ocorridos no hospital universitário do município em estudo, no ano de 2016. A amostra foi calculada com erro amostral de $10 \%$ e nível de confiança de $99 \%$. Assim, 222 usuárias foram arroladas no estudo logo após os nascimentos, contudo, após o contato para o agendamento da entrevista seis meses após o parto, para a avaliação do seguimento da criança na atenção primária, obteve-se acesso somente a 139 dessas puérperas, captadas nas diferentes regiões do município, conformando a amostra final deste estudo.

Como critérios de inclusão, as usuárias deveriam ter realizado parto no hospital universitário entre julho de 2017 e janeiro de 2018; o lactente deveria estar com seis meses de vida na data da entrevista; as usuárias deveriam ter endereço e telefone atualizados no cadastro do sistema de saúde municipal; estar na residência no dia da visita, agendada via telefone com até quatro tentativas. Foram excluídas as usuárias que não tinham a Caderneta de Saúde da Criança (CSC) no dia da entrevista; que manifestaram impeditivos de compreensão da língua portuguesa (haitianas e paraguaias, entre outras); menores de 18 anos, 
desacompanhadas de um responsável; mães de natimortos; que sofreram óbito do lactente; e, ainda, que perderam a guarda do filho.
No fluxograma (figura 1), descreve-se como aconteceu a captação, bem como as perdas da amostra do estudo.

Figura 1. Fluxograma da composição da amostra do estudo, Cascavel (PR), Brasil, 2018

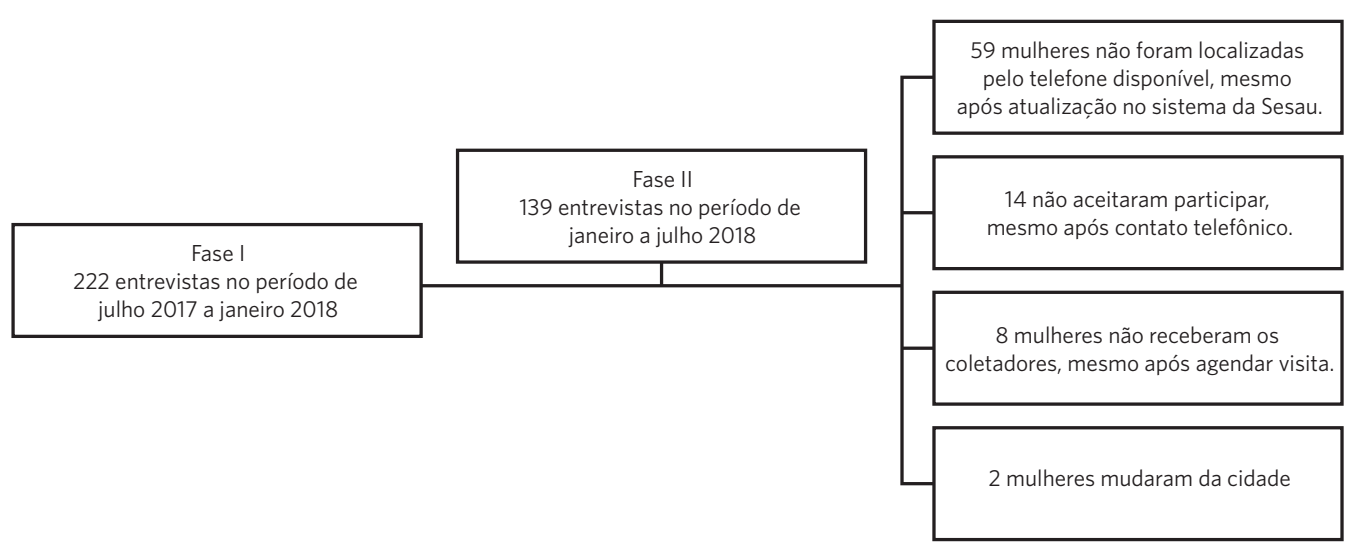

Fonte: Elaboração própria.

Para melhor compreensão da teoria, dos mecanismos de ação e das estratégias propostas pelo PRMP, criou-se o fluxograma abaixo (figura 2).

Figura 2. Teoria dos mecanismos de ação e das estratégias propostas pelo PRMP

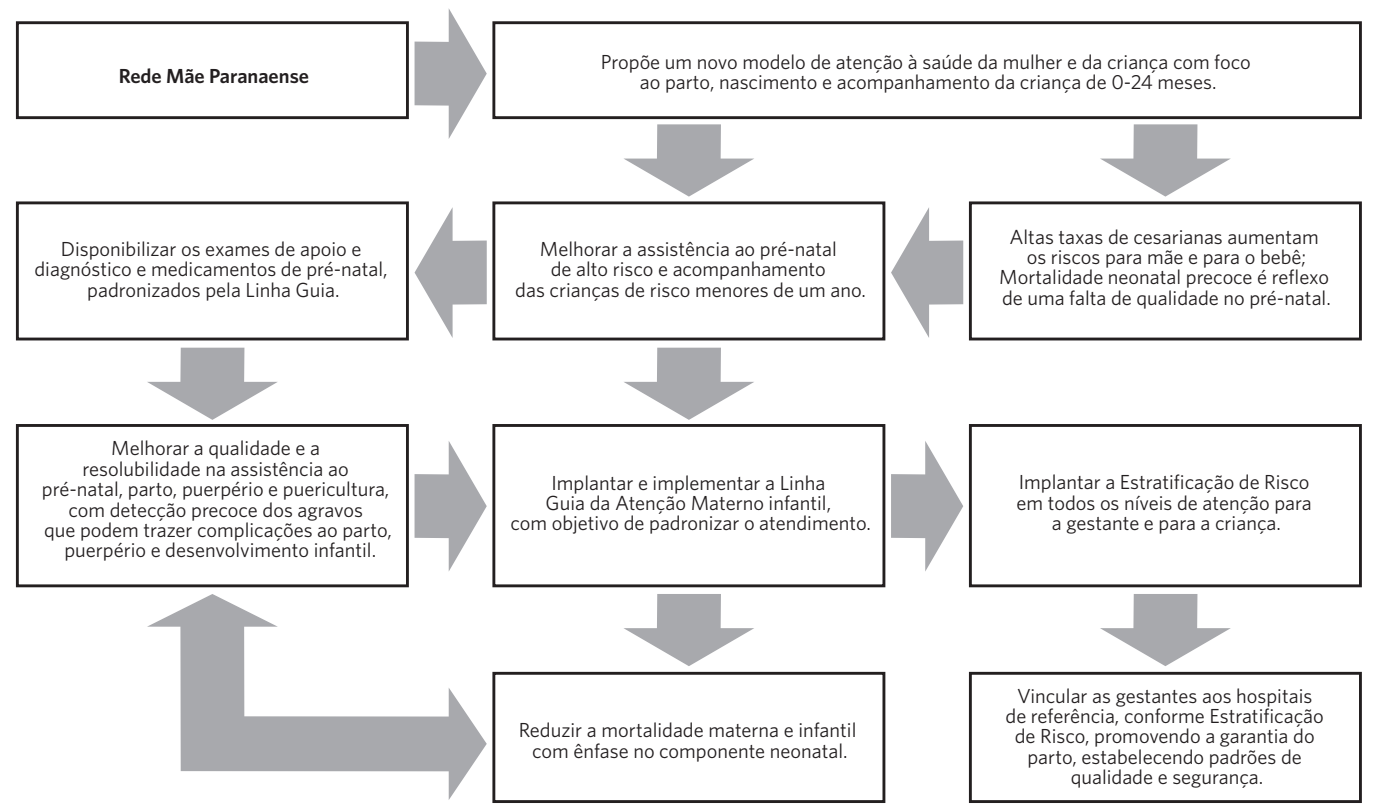

Fonte: Elaboração própria adaptada de Magalhães ${ }^{14}$. 
As entrevistas foram realizadas por pesquisadores após capacitação. Durante as visitas domiciliárias, a equipe aplicou formulário com base nos objetivos do PRMP, com questões abordando as condições clínicas maternas e da criança, aspectos sociodemográficos e dados de registros da CSC e da mãe. O referido formulário, elaborado pela equipe da pesquisa, foi testado e validado por conteúdo e aparência. Devido à deficiência de dados na CSC, em relação à estratificação de risco, houve necessidade de busca de dados das Declarações de Nascidos Vivos (DNV), encontradas nos arquivos da secretaria de saúde do município em estudo.

As variáveis analisadas, bem como os cruzamentos de análises realizados e metas propostas pelo PRMP, estão dispostos no quadro 1 .

Quadro 1. Objetivos, estratégias e ações, indicadores, meta proposta pelo PRMP e variáveis, e os cruzamentos analisados e meta do IA estipulado. Cascavel (PR), Brasil, 2018

\begin{tabular}{|c|c|c|c|c|}
\hline $\begin{array}{l}\text { Subdomínio (itens } \\
\text { do questionário da } \\
\text { pesquisa) }\end{array}$ & Indicador & Cruzamentos & Meta proposta PRMP & Fórmula \\
\hline \multicolumn{5}{|c|}{ Domínio: 1. Satisfação materna em relação ao seguimento do bebê. } \\
\hline $\begin{array}{l}\text { Número de consul- } \\
\text { tas até o sexto mês. }\end{array}$ & Número de consultas de puericultura. & $\begin{array}{l}\text { Acompanhamento na } \\
\text { puericultura. }\end{array}$ & $\begin{array}{l}100 \% \text { das crianças } \\
\text { menores de } 2 \text { anos. }\end{array}$ & $\begin{array}{l}\text { 100\% das crianças menores de } 1 \text { ano, } \\
\text { da área de abrangência, cadastradas } \\
\text { na UBS. }\end{array}$ \\
\hline $\begin{array}{l}\text { Índice de satisfação } \\
\text { das usuárias do } \\
\text { PRMP. }\end{array}$ & Satisfação da usuária. & Acesso a consultas. & $\begin{array}{l}\text { Satisfação da usuária } \\
\text { acima de } 80 \% \text {. }\end{array}$ & $\begin{array}{l}\text { - verificação do registro de nasci- } \\
\text { mento e cadastramento. }\end{array}$ \\
\hline \multicolumn{5}{|c|}{ Domínio: 2. Acompanhamento na puericultura. } \\
\hline $\begin{array}{l}\text { Itens básicos de } \\
\text { puericultura. }\end{array}$ & $\begin{array}{l}\text { PC, PT, peso, estatura, temperatura, } \\
\text { FC, FR. }\end{array}$ & $\begin{array}{l}\text { Dados verificados/da- } \\
\text { dos anotados na CSC. }\end{array}$ & $\begin{array}{l}\text { 100\% das crianças } \\
\text { acompanhadas. }\end{array}$ & $\begin{array}{l}\text { 100\% das crianças cadastradas } \\
\text { realizam primeira consulta de acom- } \\
\text { panhamento, para: } \\
\text { - avaliação da história perinatal; } \\
\text { - avaliação clínica geral; } \\
\text { - avaliação do desenvolvimento } \\
\text { neuropsicomotor; }\end{array}$ \\
\hline $\begin{array}{l}\text { Qualidade dos } \\
\text { registros. }\end{array}$ & $\begin{array}{l}\text { Dados registrados na CSC (PC, PT, } \\
\text { peso, estatura, temperatura, FC, FR). } \\
\text { Marcos do desenvolvimento neurop- } \\
\text { sicomotor (reflexos). }\end{array}$ & Registro de dados. & & $\begin{array}{l}\text { - avaliação do resultado da triagem } \\
\text { neonatal; } \\
\text { - estratificação do risco; } \\
\text { - avaliação do calendário vacinal; } \\
\text { - preenchimento da CSC; } \\
\text { - continuação do Plano de Cuidado. }\end{array}$ \\
\hline $\begin{array}{l}\text { Educação em } \\
\text { saúde. }\end{array}$ & $\begin{array}{l}\text { Presença ou ausência de orientações } \\
\text { referentes a aleitamento materno, } \\
\text { cuidados com RN/coto umbilical e } \\
\text { cuidados de higiene, prevenção de } \\
\text { acidentes domésticos (queimaduras, } \\
\text { quedas), cuidados para evitar engas- } \\
\text { gos, vacinação, introdução de ali- } \\
\text { mentação complementar, situações } \\
\text { de urgência (febre alta, engasgos, } \\
\text { traumas e ferimentos). }\end{array}$ & $\begin{array}{l}\text { Manutenção do AME; } \\
\text { Problemas de saúde; } \\
\text { Causas de atendimen- } \\
\text { tos em urgência. }\end{array}$ & $\begin{array}{l}100 \% \text { dos RN e } \\
100 \% \text { das mães da } \\
\text { área de abrangência. }\end{array}$ & $\begin{array}{l}\text { 100\% dos RN da área de abrangência } \\
\text { recebem visita domiciliar pelo enfer- } \\
\text { meiro logo após o nascimento, para: } \\
\text { - ações de educação em saúde; } \\
\text { - cadastro na puericultura; } \\
\text { - identificação de sinais de alerta. } \\
\text { 100\% das mães participam de ativi- } \\
\text { dades educativas referentes à saúde } \\
\text { infantil, conduzidas por profissional } \\
\text { de nível superior, sendo: } \\
\text { - grupos de } 20 \text { participantes; } \\
\text { - duração de uma hora; } \\
\text { - periodicidade: duas vezes/ano. }\end{array}$ \\
\hline
\end{tabular}


Quadro 1. (cont.)

Subdomínio (itens Indicador $\quad$ Cruzamentos
do questionário da
pesquisa)

pesquisa)

Domínio: 2. Acompanhamento na puericultura.

\begin{tabular}{|c|c|c|c|c|}
\hline $\begin{array}{l}\text { Consulta odonto- } \\
\text { lógica. }\end{array}$ & $\begin{array}{l}\text { Consulta agendada, consulta não } \\
\text { agendada. }\end{array}$ & $\begin{array}{l}\text { Faltou a consulta/con- } \\
\text { sulta não agendada. }\end{array}$ & $\begin{array}{l}100 \% \text { das crianças } \\
\text { acompanhadas. }\end{array}$ & $\begin{array}{l}\text { 100\% das crianças menores de } 1 \\
\text { ano realizam consulta odontológica, } \\
\text { tendo como objetivo: } \\
\text { - avaliação odontológica e plano } \\
\text { terapêutico. }\end{array}$ \\
\hline
\end{tabular}

Domínio: 3. Melhorar a qualidade e a resolubilidade na assistência ao pré-natal, parto, puerpério e à puericultura.

\begin{tabular}{|c|c|c|c|c|}
\hline $\begin{array}{l}\text { Cobertura vacinal } \\
\text { em menores de } 1 \\
\text { ano. }\end{array}$ & $\begin{array}{l}\text { Registro de vacinas na CSC (BCG, } \\
\text { Hepatite B, VIP, Pentavalente, Rotaví- } \\
\text { rus, Pneumo, Meningo, Influenza). }\end{array}$ & $\begin{array}{l}\text { Escolaridade materna; } \\
\text { Renda; } \\
\text { Orientações recebidas; } \\
\text { Busca ativa. }\end{array}$ & $\begin{array}{l}95 \% \text { cobertura va- } \\
\text { cinal. }\end{array}$ & $\begin{array}{l}\text { Número de crianças menores de } 1 \\
\text { ano vacinadas com a terceira dose da } \\
\text { Tetravalente/Pentavalente X } 100 \% \\
\text { população composta por menores } \\
\text { de } 1 \text { ano. }\end{array}$ \\
\hline $\begin{array}{l}\text { Busca ativa pela } \\
\text { unidade de saúde. }\end{array}$ & Busca ativa realizada ou não. & $\begin{array}{l}\text { Problemas de saúde } \\
\text { imunopreveníveis; }\end{array}$ & $100 \%$ busca ativa. & $\begin{array}{l}\text { Número de crianças que faltaram à } \\
\text { consulta ou vacinação e receberam } \\
\text { visita de busca ativa. }\end{array}$ \\
\hline AME. & $\begin{array}{l}\text { Avaliação do AME; Idade de manu- } \\
\text { tenção do AME; causa do desmame; } \\
\text { ajuda para AME. }\end{array}$ & $\begin{array}{l}\text { Adoecimento nas } \\
\text { crianças em AME e não } \\
\text { AME. }\end{array}$ & $80 \%$ AME. & $\begin{array}{l}\text { Taxa de prevalência de AME em } \\
\text { menores de } 4 \text { meses. }\end{array}$ \\
\hline $\begin{array}{l}\text { Transporte sani- } \\
\text { tário. }\end{array}$ & $\begin{array}{l}\text { Transporte utilizado (Samu, Siate ou } \\
\text { outros). }\end{array}$ & $\begin{array}{l}\text { Utilização de transporte } \\
\text { sanitário. }\end{array}$ & $\begin{array}{l}100 \% \text { das crianças } \\
\text { atendidas pelo trans- } \\
\text { porte sanitário. }\end{array}$ & $\begin{array}{l}\text { Porcentagem de crianças que foram } \\
\text { atendidas por transporte sanitário. }\end{array}$ \\
\hline Triagem neonatal. & $\begin{array}{l}\text { Teste do Pezinho, Teste do Olhinho, } \\
\text { Teste da Orelhinha, Teste do Cora- } \\
\text { çãozinho. }\end{array}$ & $\begin{array}{l}\text { Realização e registros } \\
\text { dos testes de triagem. }\end{array}$ & $\begin{array}{l}\text { 100\% das crianças } \\
\text { acompanhadas. }\end{array}$ & $\begin{array}{l}\text { 100\% dos RN realizam o Teste do } \\
\text { Pezinho após } 48 \text { horas de nascidos, } \\
\text { bem como os testes da Orelhinha, do } \\
\text { Olhinho e do Coraçãozinho. }\end{array}$ \\
\hline \multicolumn{5}{|c|}{ Domínio: 4. Estratificação de risco em todos os níveis de atenção para a gestante e para a criança. } \\
\hline $\begin{array}{l}\text { Porcentagem de } \\
\text { crianças estratifi- } \\
\text { cadas como de alto } \\
\text { risco, de acordo } \\
\text { com os critérios es- } \\
\text { tabelecidos na linha } \\
\text { guia do PRMP. }\end{array}$ & $\begin{array}{l}\text { Criança classificada; } \\
\text { Criança não classificada. }\end{array}$ & $\begin{array}{l}\text { Encaminhamento para } \\
\text { ambulatório especiali- } \\
\text { zado para alto risco. }\end{array}$ & $\begin{array}{l}100 \% \text { das crianças } \\
\text { estratificadas. }\end{array}$ & $\begin{array}{l}\text { 100\% das crianças cadastradas } \\
\text { realizam estratificação de risco na } \\
\text { primeira consulta. } \\
\text { OBS: a estratificação de risco deve } \\
\text { ser realizada na primeira visita do- } \\
\text { miciliar e em todas as consultas e } \\
\text { visitas subsequentes. } \\
100 \% \text { das crianças de risco habitual } \\
\text { cadastradas realizam sete consultas } \\
\text { subsequentes à primeira consulta, no } \\
\text { primeiro ano de vida. } \\
\text { 100\% das crianças de risco inter- } \\
\text { mediário cadastradas realizam oito } \\
\text { consultas subsequentes à primeira } \\
\text { consulta, no primeiro ano de vida. } \\
\text { 100\% das crianças de alto risco } \\
\text { cadastradas realizam nove consultas } \\
\text { subsequentes à primeira consulta, no } \\
\text { primeiro ano de vida. }\end{array}$ \\
\hline
\end{tabular}

RN=Recém-Nascido; PC=Perímetro Cefálico; PT=Perímetro Torácico; FC=Frequência Cardíaca; FR=Frequência Respiratória; BCG=Bacillus Calmette-Guérin; VIP=Vacina Inativada Poliomielite; AME=Aleitamento Materno Exclusivo; UBS=Unidade Básica de Saúde; Samu=Serviço de Atendimento Móvel de Urgência; Siate=Serviço Integrado de Atendimento ao Trauma em Emergência. 
Os dados coletados durante as entrevistas e a análise dos dados da CSC foram tabulados em planilhas do programa Microsoft Excel ${ }^{\circledR}$, com conferência dupla de digitadores.

Para tanto, com base na compreensão dos mecanismos e das estratégias propostas pelo PRMP, buscou-se, diante da diversidade de respostas das participantes, analisar as variáveis de acordo com os objetivos do PRMP, estabelecendo um IA para cada objetivo, para posterior comparação com a proposta inicial de objetivos e metas do PRMP.

O IA foi construído pela seleção das variáveis relativas às metas da matriz da linha guia - que descreve os objetivos estratégicos do PRMP -, denominadas no quadro 1 como indicadores. Assim, a partir dos objetivos estratégicos dispostos, foram criados quatro domínios para agrupar as variáveis que possibilitaram a criação do IA (quadro 1), visto que, na linha guia do PRMP, constavam o objetivo estratégico e a porcentagem para o alcance da meta, sem a descrição de quais indicadores compunham cada objetivo. Cada domínio era composto por subdomínios, como descrito no quadro 1, os quais se referiam aos dados coletados pelos pesquisadores com instrumento elaborado para investigação.

Uma vez selecionadas as variáveis, e organizadas em seus respectivos domínios, suas frequências relativas observadas foram selecionadas e comparadas com as frequências relativas esperadas, definidas nas metas do PRMP. Para avaliar quanto da meta proposta pelo programa foi alcançado no município em estudo, calculou-se o IA mediante razão da somatória das frequências observadas nos dados coletados e somatório das frequências propostas pelo PRMP. O mesmo procedimento foi também realizado para cada um dos domínios avaliados, calculando a razão do somatório das frequências relativas observadas e o somatório das frequências relativas esperadas (frequência absoluta) de cada domínio, para assim identificar os principais problemas relativos ao não atingimento da meta plena. Foram consideradas frequências esperadas equivalentes a $100 \%$ para os itens: número de consultas realizadas e registradas na CSC; avaliação clínica, avaliação do resultado dos testes; avaliação do calendário vacinal; preenchimento de dados da CSC (Perímetro Cefálico - PC, peso, estatura, resultados dos testes de triagem); realização de visita domiciliar; participação em atividades educativas e realização de consulta odontológica; registro de terceira dose da vacina Tetravalente/ Pentavalente na CSC; busca ativa a faltosos; taxa de Aleitamento Materno Exclusivo (AME) aos 4 meses e realização dos testes de triagem neonatal (testes do Pezinho, da Orelhinha, do Olhinho e do Coraçãozinho); estratificação de risco. Para o domínio 'Satisfação Materna', a frequência esperada foi de $80 \%$, considerando as metas propostas pelo PRMP.

As participantes da pesquisa foram esclarecidas, com relação ao objetivo, da necessidade de concessão de nova entrevista, no sexto mês após a alta, por meio de visita domiciliar, agendada por contato telefônico, sobre o seguimento da criança no PRMP. Elas anuíram sua participação na pesquisa com a assinatura do Termo de Consentimento Livre e Esclarecido.

Trata-se uma pesquisa multicêntrica, que foi analisada e aprovada pelo Comitê de Ética da Universidade Estadual de Londrina (CEP/ UEL), sob parecer $\mathrm{n}^{\mathrm{0}} 2.053 .304$.

\section{Resultados}

A amostra do estudo se caracterizou por mulheres com idades entre 17 e 43 anos, que, em sua maioria, residiam com três a cinco outros moradores, sendo que tais domicílios apresentavam entre três e seis cômodos, em média. A maioria dos casais tinha um ou dois filhos ( $n=96 ; 69 \%)$, sendo que a maior parte das usuárias tinha completado o ensino médio ( $\mathrm{n}=55 ; 39,5 \%$ ), tinham ocupação remunerada $(\mathrm{n}=84 ; 60,4 \%)$ e não recebia nenhum benefício de programas de transferência de renda $(n=128 ; 92 \%)$. Por fim, quanto à escolaridade paterna, a maior parte apresentou ensino médio completo ( $n=37 ; 26,6 \%)$. Estes dados são observados na tabela 1 . 
Tabela 1. Demonstrativo das variáveis socioeconômicas. Cascavel (PR), Brasil $(n=139)$

\section{Características}

sociodemográficas

Idade materna

\section{Categoria}

17-20 anos

21-24 anos

25-28 anos

29-32 anos

33-36 anos

37-40 anos

41-43 anos

Raça $\quad$ Branca 101

\begin{tabular}{ll} 
& Negra \\
& Parda \\
& Amarela \\
& Sem registro \\
\hline Situação conjugal & Com companheiro \\
& Sem companheiro \\
\hline Número de moradores na & 2 \\
& 3 \\
& 4 \\
& 5 \\
& 6 \\
& 7
\end{tabular}

\begin{tabular}{ll}
\hline Número de filhos & 1 \\
& 3 \\
3 \\
4 \\
5 \\
6
\end{tabular}

\begin{tabular}{|c|c|c|c|}
\hline \multirow[t]{6}{*}{ Escolaridade materna } & Fundamental completo & 17 & 12,2 \\
\hline & Fundamental incompleto & 33 & 23,7 \\
\hline & Médio completo & 55 & 39,5 \\
\hline & Médio incompleto & 18 & 12,9 \\
\hline & Superior completo & 8 & 5,7 \\
\hline & Superior incompleto & 8 & 5,7 \\
\hline \multirow[t]{5}{*}{ Escolaridade paterna } & Fundamental completo & 26 & 18,7 \\
\hline & Fundamental incompleto & 36 & 25,9 \\
\hline & Médio completo & 37 & 26,6 \\
\hline & Médio incompleto & 17 & 12,2 \\
\hline & Superior completo & 3 & 2,1 \\
\hline
\end{tabular}

Frequência $\quad \%$

15,1

25,9

25,1

15,1

10,8

4,3

3,6

\begin{tabular}{rr}
5 & 3,6 \\
\hline 101 & 72,6
\end{tabular}

$5 \quad 3,6$

$30 \quad 21,5$

$2 \quad 1,4$

$1 \quad 0,7$

2791,3

$12 \quad 8,6$

42,8

$39 \quad 28$

$47 \quad 33,8$

$31 \quad 22,3$

$10 \quad 7,1$

$4 \quad 2,8$

$4 \quad 2,8$

$48 \quad 34,5$

$48 \quad 34,5$

$30 \quad 21,5$

$8 \quad 5,7$

42,8

$1 \quad 0,7$




\begin{tabular}{|c|c|c|c|}
\hline $\begin{array}{l}\text { Características } \\
\text { sociodemográficas }\end{array}$ & Categoria & Frequência & $\%$ \\
\hline \multirow[t]{3}{*}{ Escolaridade paterna } & Superior incompleto & 3 & 2,1 \\
\hline & Não sabe informar & 12 & 8,6 \\
\hline & Sem registro & 5 & 3,6 \\
\hline \multirow[t]{3}{*}{ Ocupação materna } & Remunerada & 84 & 60,4 \\
\hline & Não remunerada & 54 & 38,8 \\
\hline & Sem registro & 1 & 0,7 \\
\hline \multirow[t]{11}{*}{ Renda } & Sem renda & 1 & 0,7 \\
\hline & $<900$ reais & 1 & 0,7 \\
\hline & $900-1.500$ & 35 & 25,1 \\
\hline & $1.600-2.500$ & 43 & 30,9 \\
\hline & $2.600-3.800$ & 29 & 20,8 \\
\hline & $4.000-4.800$ & 10 & 7,2 \\
\hline & $5.000-6.000$ & 4 & 2,8 \\
\hline & $6.000-9.000$ & 1 & 0,7 \\
\hline & Não informou & 2 & 1,4 \\
\hline & Não sabe & 5 & 3,5 \\
\hline & Sem registro & 8 & 5,7 \\
\hline \multirow[t]{3}{*}{ Bolsa Família } & Sim & 8 & 5,7 \\
\hline & Não & 128 & 92 \\
\hline & Sem registro & 3 & 2,1 \\
\hline
\end{tabular}

Fonte: Elaboração própria. Instrumento coleta de dados pesquisa, Cascavel (PR), Brasil, 2018.

Os itens avaliados para cálculo do IA basearam-se na matriz proposta pelo PRMP para monitoramento da implementação deste nas regionais e nos municípios do Estado, e estão descritos na tabela 2.

Em relação ao primeiro objetivo relacionado ao domínio ‘Satisfação Materna', especificamente no que se refere ao seguimento da criança, foram avaliados os subitens: número de consultas realizadas e registradas na CSC, bem como a satisfação materna em relação ao atendimento. Foi evidenciada uma frequência relativa de $68 \%$ de satisfação das usuárias.

No segundo objetivo, que se refere ao domínio 'Acompanhamento na Puericultura', foram utilizados os parâmetros adotados pelo PRMP em relação à avaliação clínica, avaliação do resultado dos testes, avaliação do calendário vacinal, ao preenchimento de dados da CSC (PC, peso, estatura, resultados dos testes de triagem), à realização de visita domiciliar, participação em atividades educativas e realização de consulta odontológica. Esse objetivo alcançou um IA de $67 \%$.

No terceiro objetivo, caracterizado pelo domínio 'Qualidade e Resolubilidade na Assistência', foram avaliados o registro de terceira dose da vacina Tetravalente/ Pentavalente na CSC, a busca ativa a faltosos, a taxa de AME aos 4 meses e a realização dos testes de triagem neonatal (testes do Pezinho, da Orelhinha, do Olhinho e do Coraçãozinho), sendo possível identificar um IA de apenas $55 \%$. 
Por fim, o quarto objetivo condizente com o domínio 'Estratificação de Risco', identificou que o IA foi de apenas $6 \%$, evidenciando-se, portanto, índices insatisfatórios entre os domínios analisados. A estratificação de risco não está acontecendo de maneira sistemática, o que prejudica os encaminhamentos e o seguimento adequado da criança.

Tabela 2. IA em relação às metas alcançadas conforme os objetivos PRMP, Cascavel (PR), Brasil, 2018

\begin{tabular}{|c|c|c|c|c|}
\hline \multirow[t]{3}{*}{ Domínios } & \multirow[t]{3}{*}{ Proposta } & \multicolumn{2}{|c|}{ Frequência da meta } & \multirow{3}{*}{$\begin{array}{l}\text { IA por } \\
\text { domínio }\end{array}$} \\
\hline & & Proposta & Observada & \\
\hline & & PRMP (FP) & estudo (FO) & \\
\hline \multirow[t]{2}{*}{ 1. Satisfação Materna } & $\begin{array}{l}\text { 1.1 Atendimento até o sexto mês (pelo menos cinco } \\
\text { consultas); }\end{array}$ & 1,00 & 0,4964 & $68 \%$ \\
\hline & 1.2 Satisfação. & 0,80 & 0,7266 & \\
\hline \multirow{10}{*}{$\begin{array}{l}\text { 2. Acompanhamento } \\
\text { na Puericultura }\end{array}$} & 2.1 Avaliação clínica geral (exame físico); & 1,00 & 0,6691 & $67 \%$ \\
\hline & 2.2 Avaliação do resultado da Triagem Neonatal & 1,00 & 0,4748 & \\
\hline & 2.3 Avaliação do calendário vacinal; & 1,00 & 0,5468 & \\
\hline & 2.4 Preenchimento da CSC - Peso; & 1,00 & 0,9060 & \\
\hline & 2.5 Preenchimento da CSC - Estatura; & 1,00 & 0,8920 & \\
\hline & 2.6 Preenchimento da CSC - PC; & 1,00 & 0,9140 & \\
\hline & 2.7 Preenchimento da CSC - testes; & 1,00 & 0,4748 & \\
\hline & 2.8 Visita domiciliar logo após o nascimento; & 1,00 & 0,5396 & \\
\hline & 2.9 Mães participam de atividades educativas; & 1,00 & 0,0144 & \\
\hline & 2.10 Consulta odontológica em menores de 1 ano. & 1,00 & 0,3166 & \\
\hline \multirow{7}{*}{$\begin{array}{l}\text { 3. Qualidade e Resolu- } \\
\text { bilidade na } \\
\text { Assistência }\end{array}$} & $\begin{array}{l}\text { 3.1 Número de crianças menores de } 1 \text { ano vacinadas } \\
\text { com a terceira dose da Tetravalente/Pentavalente; }\end{array}$ & 1,00 & 0,5468 & $55 \%$ \\
\hline & 3.2 Busca ativa a faltosos; & 1,00 & 0,0647 & \\
\hline & $\begin{array}{l}\text { 3.3 Taxa de prevalência de AME em menores de } 4 \\
\text { meses; }\end{array}$ & 1,00 & 0,5827 & \\
\hline & 3.4 Teste do Pezinho; & 1,00 & 0,9856 & \\
\hline & 3.5 Teste da Orelhinha; & 1,00 & 0,9496 & \\
\hline & 3.6 Teste do Olhinho; & 1,00 & 0,9568 & \\
\hline & 3.7 Teste do Coraçãozinho. & 1,00 & 0,9712 & \\
\hline $\begin{array}{l}\text { 4. Estratificação de } \\
\text { Risco }\end{array}$ & 4.1 Estratificação do Risco. & 1,00 & 0,0576 & $6 \%$ \\
\hline \multirow[t]{2}{*}{ IA Geral } & Somatório. & 19,80 & 12,0860 & \\
\hline & Proporção sobre a meta. & & & $61 \%$ \\
\hline
\end{tabular}

Fonte: Elaboração própria, com os dados da pesquisa multicêntrica 2017-2018.

\section{Discussão}

O IA obtido na análise dos dados será discutido em relação a cada domínio estabelecido no estudo.

\section{Satisfação Materna}

O PRMP propõe que $100 \%$ das crianças sejam acompanhadas até o sexto mês pelas unidades de saúde e almeja que $80 \%$ das 
usuárias-mães dessas crianças estejam satisfeitas com a assistência prestada. No entanto, os dados encontrados apontaram que 49,6\% $(\mathrm{FO}=0,4964)$ das crianças em estudo foram acompanhadas e a satisfação materna foi de $72,6 \%$ (FO=0,7266), obtendo-se, assim, um IA no domínio 'Satisfação Materna' de $68 \%$. O vínculo estabelecido entre a equipe e a família se configura, em tecnologia leve na atenção primária, como ferramenta primordial para a construção do plano de cuidados e, por conseguinte, para uma adesão maior aos programas propostos pela unidade de saúde, bem como aos tratamentos indicados.

O IA encontrado demonstra que a meta estabelecida pelo PRMP não foi atingida. Assim, o vínculo e o acesso ao serviço impossibilitam a adesão adequada ao seguimento da criança. Estabelecer vínculo entre a equipe e a família, promovendo o apoio profissional às famílias, aumenta as chances de sucesso no cuidado do recém-nascido ${ }^{\mathbf{1 5 , 1 6}}$. Contudo, mesmo sendo ofertado o acesso pela disponibilização dos atendimentos, o agendamento de consultas programadas é um dos fatores que interferem na adesão e participação das mães nas consultas de puericultura. Constata-se que falta de vínculo entre profissional e família compromete a longitudinalidade do cuidado à criança ${ }^{17}$, apontando para uma menor busca pelo serviço para o seguimento da criança após o parto.

Assim, percebe-se que, apesar de a maioria das mães ter relatado satisfação em relação ao atendimento, a adesão às consultas não acontece conforme o preconizado, observando-se, em média, menos de cinco consultas realizadas até o sexto mês de vida. Diante desse cenário, a partir da frequência observada $(0,4964)$ dessas consultas, foi verificado que, nesse aspecto, o PRMP é considerado insatisfatório, o que também foi observado em estudo realizado ${ }^{18}$ no município de Palmital (PR). Tem-se, portanto, um paradoxo em que, mesmo tendo as mães relatado satisfação com o atendimento prestado, elas ainda não estão comparecendo em todas as consultas agendadas, ou ainda, verifica-se que as consultas não estão sendo agendadas/programadas conforme preconiza o PRMP. Esta situação põe em risco a saúde das crianças, visto que o acompanhamento mais frequente nos primeiros meses de vida é considerado o período ‘ouro' para a detecção precoce de anormalidades, bem como para o encaminhamento de intervenções que, se não realizadas, podem comprometer $o$ desenvolvimento da criança de maneira irreversível ${ }^{18}$. Para tanto, se faz necessário um esforço maior das equipes de atenção primária, no intuito de sensibilizar as mães a comparecerem às consultas e, ainda, na organização do processo de trabalho, a fim de garantir o número de consultas adequadas, conforme o preconizado para garantir a qualidade da atenção prestada à criança.

\section{Acompanhamento na Puericultura}

Em relação ao objetivo acompanhamento da criança na puericultura, as mães eram questionadas sobre os procedimentos realizados durante a consulta de puericultura. Com base nesses dados, evidenciou-se que o profissional realizava os procedimentos inerentes a esse domínio, mas, na maioria das vezes, não realizava o registro adequado na CSC, o que trouxe limitações ao presente estudo. Em estudo qualitativo com profissionais médicos, estes relatam que encontram dificuldades na manipulação da CSC, e que esta não facilita o atendimento ${ }^{10}$. Em estudo ${ }^{19}$ realizado no Recife (PE), no entanto, verificou-se que todos os enfermeiros fizeram os registros das medidas antropométricas (peso, estatura, PC), isto se contrapondo ao que foi aqui identificado, quando ainda se percebem falhas de registro, principalmente em relação ao preenchimento dos resultados dos testes do Pezinho com registro inferior a $50 \%(\mathrm{FO}=0,4748)$, apesar de terem sido realizados na maioria das crianças acompanhadas $(\mathrm{FO}=0,9856)$.

Pressupõe-se que os resultados não estão sendo avaliados pelo profissional, o que descaracteriza o objetivo primordial dos testes de triagem neonatal, que é a captação precoce das 
crianças com doenças detectáveis por esses testes. Os dados relativos às medidas da criança (peso, estatura e $\mathrm{PC}$ ) foram mais satisfatórios $(\mathrm{FO}=0,9060 ; \mathrm{FO}=0,8920 ; \mathrm{FO}=0,9140)$, demonstrando maior frequência de registros. Detectouse, ainda, frequência observada insatisfatória em relação à realização da visita domiciliar $(\mathrm{FO}=0,5396)$, ações educativas $(\mathrm{FO}=0,0144)$ e consulta odontológica $(\mathrm{FO}=0,3166)$, refletindo em assistência baseada ainda no modelo tradicional, pouco voltada à prevenção. O PRMP tem como meta $100 \%$ de alcance para todos estes quesitos, contudo, os dados do presente estudo demonstram IA de $67 \%$.

Na busca de uma real mudança no modelo assistencial de saúde vigente, a atenção primária como coordenadora do cuidado torna-se um pilar estruturante deste processo. Para tanto, deveria voltar-se a uma assistência embasada na prevenção de agravos, com o envolvimento de ações educativas na rotina dos profissionais, visando à promoção do autocuidado. Outro aspecto relevante é o desenvolvimento de habilidades no cuidador, pois fortalece as estratégias domiciliárias para o cuidado adequado com a criança, e também promove um espaço para sanar dúvidas, ensinar o cuidado e agir com segurança e harmonia, buscando a superação das dificuldades relacionadas ao despreparo ${ }^{15,16}$. Evidencia-se, assim, que esta prática deve ser incluída no plano de cuidados e desenvolvida de maneira rotineira, visando a uma atenção integral e longitudinal, superando os desafios no âmbito da saúde da criança.

\section{Qualidade e Resolubilidade na Assistência}

Em relação à qualidade da assistência, os princípios de redução dos indicadores de morbimortalidade infantil, vacinação total da população e manutenção do aleitamento materno são práticas que evitariam milhares de mortes em crianças menores de cinco anos'.

Referente à cobertura vacinal, o indicador é insatisfatório $(\mathrm{FO}=0,5468)$, o que tem causado preocupação em relação ao ressurgimento de doenças e, ainda, mortes evitáveis, principalmente em menores de 1 ano. Evidenciada em estudo realizado em Minas Gerais ${ }^{20}$ e corroborada pelos presentes dados, verificou-se a elevação do número de casos de coqueluche, entre 2015 (38 casos confirmados) e 2017 (42 casos suspeitos e 2 confirmados), e em 2018 , no período de janeiro a setembro, quando houve 7 casos confirmados ${ }^{21}$. Uma estratégia que poderia melhorar esse indicador seria a busca ativa pela unidade de saúde da atenção primária, mas esta é pouco utilizada pelas equipes $(\mathrm{FO}=0,0647)$, contrapondo-se ao preconizado nas metas do PRMP.

Em relação ao aleitamento materno, a Organização Mundial da Saúde (OMS) e o Ministério da Saúde recomendam o AME até o sexto mês. Porém, como parâmetro avaliativo, o PRMP utiliza a cobertura de $100 \%$ de AME até o quarto mês de vida. Mesmo diante de sua comprovada importância, o aleitamento materno ainda apresenta indicadores insatisfatórios $(\mathrm{FO}=0,5827)$, quando comparados com estudo desenvolvido em Portugal, no qual a taxa de manutenção do aleitamento materno aos 4 meses foi de $78,7 \%^{\mathbf{2 2}}$. No entanto, mesmo a baixa adesão ainda foi maior do que as taxas apresentadas em estudos brasileiros realizados em Uberlândia (MG) ${ }^{\mathbf{2 3}}$ (50,6\%), Porto Alegre (RS) ${ }^{\mathbf{2 4}}$ (47,1\%) e Londrina (PR) ${ }^{\mathbf{2 5}}$ (53,7\%). Neste sentido, o aconselhamento e apoio às mães e à família, nos primeiros meses de vida, se tornam fundamentais, formando uma rede de apoio que pode auxiliar na superação das dificuldades e dúvidas comuns desta fase ${ }^{26}$.

Outro indicador para avaliar a qualidade e resolubilidade na assistência são os testes de triagem neonatal. Com o estudo, constatou-se que os testes foram realizados na maioria das crianças $(\mathrm{FO}=0,9856 ; \mathrm{FO}=3.5=0,9496$; $\mathrm{FO}=0,9568 ; \mathrm{FO}=0,9712)$, mesmo quando não estavam registrados no prontuário. Porém, quando houve falhas em algum equipamento, alguns testes acabaram não sendo realizados, e isso gerou queixas das mães, pois algumas relataram que não foram informadas quando os equipamentos voltaram a funcionar, ficando, 
assim, sem realizar os testes e comprometendo possíveis diagnósticos precoces.

Portanto, o IA identificado no presente estudo, para o domínio 'Qualidade e Resolubilidade na Assistência', foi de 55\% alcançados entre as usuárias investigadas, demonstrando fragilidades nas estratégias de prevenção e captação precoce de doenças em crianças, o que pode comprometer a integralidade do cuidado, um dos princípios da atenção primária. Deste modo, se faz necessário desfragmentar o cuidado, melhorando a responsabilização e o compromisso dos profissionais, indo ao encontro de uma assistência cada vez mais qualificada e resolutiva.

\section{Estratificação de Risco}

O PRMP estabelece a estratificação de risco para $100 \%$ das gestantes e crianças, como elemento orientador para a organização da rede em seus diversos níveis de atenção. A estratificação de risco é utilizada no manejo das condições crônicas e dos dados epidemiológicos, permitindo a organização dos serviços para oferecer o recurso assistencial mais adequado ${ }^{5}$.

No contexto do estudo realizado, esse domínio foi o que recebeu menor pontuação, pois, na maioria das CSC, não está registrada a estratificação de risco da criança. A despeito de sua importância, não tem sido vista como prioridade na condução da puericultura e nos encaminhamentos realizados pela equipe. Apesar de o risco intermediário ter sido considerado como diferencial no Estado do Paraná, diante do Rede Cegonha, os profissionais não incorporam esta prática em seu cotidiano de trabalho ${ }^{\mathbf{1 0}}$.

Frente ao IA de $6 \%$ detectado neste estudo, não se tem parâmetros para avaliar como estão acontecendo os acompanhamentos, de acordo com a classificação risco habitual, intermediário e alto risco. Desse modo, houve a necessidade de busca manual nos arquivos do Programa Ninar do município em estudo, o qual tem o objetivo de reduzir o índice de morbimortalidade infantil, por meio do desenvolvimento de ações técnicas, capazes de identificar e intervir nos fatores de risco. Obtiveram-se informações nas Declarações de Nascidos Vivos dos 139 recém-nascidos da amostra e fez-se a classificação de risco dessas crianças, seguindo as diretrizes propostas no PRMP. Constatou-se que 106 (76,2\%) foram classificadas como de risco habitual; 12 (8,6\%), como risco intermediário; e 21 (15,1\%), como alto risco. Entretanto, não foi possível analisar se essas crianças de risco intermediário e alto risco foram posteriormente acompanhadas em ambulatórios especializados, pois não foram encontrados registros nas CSC durante a entrevista com as usuárias. A não realização da estratificação de risco (ou a classificação inadequada da criança) pode ocasionar consultas desnecessárias para o risco habitual e até mesmo seguimento inadequado das crianças de risco intermediário e alto risco. Desta forma, compromete-se o seguimento da criança após o parto, o qual vislumbra o desenvolvimento e crescimento adequado de todas as crianças nascidas e atendidas pelo PRMP.

\section{Considerações finais}

O desenvolvimento do presente estudo possibilitou o estabelecimento de índices avaliativos para cada objetivo do PRMP. Oito anos após a sua implantação, podem-se constatar os pontos fortes em relação à satisfação materna, importante indicador para o estabelecimento de estratégias para os profissionais de saúde que atuam junto aos usuários. Os testes de triagem estão sendo realizados na maioria das crianças, refletindo uma melhoria na qualidade da assistência prestada e detecção precoce de doenças. Em contrapartida, o PRMP ainda apresenta pontos que necessitam ser fortalecidos, principalmente em relação à vacinação, pois o ressurgimento de doenças já erradicadas mostra que a falha vacinal nas crianças tem como impacto o surgimento de epidemias. 
Outro ponto a ser considerado é a falha no registro das atividades dos enfermeiros, o que não permite que outros profissionais possam comparar os dados anteriores com os dados atuais da criança, assim dificultando o seguimento. Em relação à estratificação de risco, vista como um diferencial no PRMP, pelo fato de organizar a assistência e direcionar as condutas e práticas, constatou-se que não está sendo realizada de maneira uniforme no município do estudo, dificultando os direcionamentos no seguimento da criança de risco pelo PRMP.

Dessa maneira, entende-se que, para se trabalhar em rede, é fundamental que todos os pontos de atenção necessários ao cuidado materno-infantil estejam conectados, para o seu bom funcionamento, minimizando, assim, a fragmentação, o desperdício de recursos e a ineficiência. Espera-se que esses dados contribuam e instiguem novos estudos sobre a temática, sempre buscando garantir a integralidade da assistência e um crescimento e desenvolvimento saudável às crianças.

\section{Colaboradores}

Santos DR (0000-0002-3521-7272)*, Viera CS (0000-0002-0900-4660)* e Ferrari RAP (00000003-0157-7461)* contribuíram para a concepção e delineamento do estudo; a aquisição, análise e interpretação dos dados do trabalho; elaboração de versões preliminares do artigo e revisão crítica de importante conteúdo intelectual; aprovação final da versão a ser publicada; concordância em ser responsável por todos os aspectos do trabalho, no sentido de garantir que as questões relacionadas à exatidão ou à integridade de qualquer parte da obra sejam devidamente investigadas e resolvidas. Guimarães ATB (00000002-3633-6484)* contribuiu para a concepção e delineamento do estudo; a aquisição, análise e interpretação dos dados do trabalho; elaboração de versões preliminares do artigo e revisão crítica de importante conteúdo intelectual; aprovação final da versão a ser publicada. Toso BRGO (00000001-7366-077X)* contribuiu para revisão crítica de importante conteúdo intelectual; aprovação final da versão a ser publicada.

\section{Referências}

1. Victora CGV, Aquino EML, Leal MC, et al. Saúde de mães e crianças no Brasil: progressos e desafios. Saúde no Brasil [internet]. 2011 [acesso em $2018 \mathrm{fev}$ 17]; 2:32-46. Disponível em: http://bvsms.saude.gov.br/ bvs/artigos/artigo_saude_brasil_2.pdf.

2. Diniz CSG. Gênero, saúde materna e o paradoxo perinatal. Rev. Brasileira de Crescimento e Desenvolvimento Humano [internet]. 2009 [acesso em 2018 set 12]; 19(2):313-326. Disponível em: http://www.
producao.usp.br/handle/BDPI/14348.

3. Cassiano ACM, Carlucci EMS, Gomes CF, et al. Saúde materno-infantil no Brasil: evolução e programas desenvolvidos pelo Ministério da Saúde. Rev. Serv. Públ. [internet]. 2014 [acesso em 2018 set 16]; 65(2):227-244. Disponível em: https://revista.enap. gov.br/index.php/RSP/article/view/581/499.

4. Restrepo-Méndez MC, Barros AJ, Requejo J, et al.
*Orcid (Open Researcher and Contributor ID). 
Progress in reducing inequalities in reproductive, maternal, newborn and child health in Latin America and the Caribbean: an unfinished agenda. Revista Panamericana de Salud Pública [internet]. 2015 [acesso em 2018 ago 2]; 38(1):9-16. Disponível em: https:// www.scielosp.org/pdf/rpsp/2015.v38nl/09-16/en.

5. Paraná. Secretaria de Estado da Saúde, Superintendência de Atenção à Saúde. Linha Guia da Rede Mãe Paranaense. Paraná: Sesa, 2018. [acesso em 2018 jul 30]. Disponível em: http://www.saude.pr.gov.br/arquivos/File/LinhaGuiaMaeParanaense_2018.pdf.

6. Frank BRB, Toso BRGDO, Viera CS, et al. Avaliação da implementação da Rede Mãe Paranaense em três Regionais de Saúde do Paraná. Saúde debate [internet]. 2016 [acesso em 2017 jun 5]; 40(109):163-174. Disponível em: https://doi.org/10.1590/0103-1104201610913.

7. Baggio MA, Pereira FDC, Guimarães ATB, et al. Programa Rede Mãe Paranaense: análise da atenção pré-natal em uma regional de saúde. Cogitare Enferm. [internet]. 2016 [acesso em $2017 \mathrm{dez} 20$ ]; 21(3):1-10. Disponível em: http://www.redalyc.org/articulo. oa? id $=483653826007$.

8. Netto A, Silva RMMD, Santos MF, et al. Mortalidade Infantil: avaliação do Programa Rede Mãe Paranaense em regional de saúde do Paraná. Cogitare Enferm. [internet]. 2017 [acesso em $2018 \mathrm{fev}$ 6]; 22(1):01-08. Disponível em: http://www.redalyc.org/ articulo.oa?id=483654742008.

9. Demiti JMG, Gasquez ADSA. Rede Mãe Paranaense: análise comparativa da mortalidade materno infantil entre estado e município. Revista Uningá Review [internet]. 2018 [acesso em 2018 out 15]; 30(1):6-10. Disponível em: http://revista.uninga.br/index.php/ uningareviews/article/view/2006/1600.

10. Rocha RRM, Caldeira S, França AFO, et al. Percepção de médicos sobre a implantação e desenvolvimento do programa rede mãe paranaense. Rev. Pesq. Qualit. [internet]. 2017 [acesso em 2017 nov 5]; 5(7):143167. Disponível em: https://editora.sepq.org.br/index. php/rpq/article/view/48/69.
11. Rocha RRM, Ferreira AFO, Zilly A, et al. Conhecimento e perspectiva de enfermeiros na rede de atenção materna e infantil do Paraná. Ciência, Cuidado e Saúde [internet]. 2018 [acesso em 2018 jul 14]; 17(1):17. Disponível em: http://ojs.uem.br/ojs/index.php/ CiencCuidSaude/article/view/39235/pdf.

12. Arretche M. Avaliação de políticas públicas é objeto de pesquisa [internet]. Comciencia, SBPC/Labjor. 2002 [acesso em 2018 jul 15]. Disponível em: http:// www.comciencia.br/dossies-1-72/entrevistas/ppublicas/arretche.htm.

13. Prefeitura Municipal de Cascavel, Secretaria Municipal de Saúde [internet]. [acesso em 2019 set 15]. Disponível em: https://cascavel.atende. net/?pg=subportal\&chave=13\#!/tipo/pagina/valor $/ 225$.

14. Magalhães R. Implementação de programas multiestratégicos: uma proposta de matriz avaliativa. Ciênc. Saúde Colet. [internet]. 2014 [acesso em 2018 nov 8]; 19(7):2115-2123. Disponível em: https://www.scielosp. org/pdf/cartão/2014.v19n7/2115-2123.

15. Brambila ILM, Oliveira TP, Silva JM, et al. O cuidado domiciliário ao recém-nascido de risco no primeiro ano de vida: experiência dos pais. Rev. Diálogos \& Saberes [internet]. 2016 [acesso em $2018 \mathrm{fev} 12$ ]; 11(1):7392. Disponível em: http://www.fafiman.br/seer/index.php/dialogosesaberes/article/view/367/350.

16. Lopes KDCL, Oliveira Neto JGD, Sá GGDM, et al. Dificuldades nos cuidados ao recém-nascido: realidades de puérperas primíparas. Rev. Saúde Púb. Santa Catarina [internet]. 2015 [acesso em 2017 out 2]; 8(3):19-33. Disponível em: http://revista.saude.sc.gov. br/index.php/inicio/article/view/351/317.

17. Reichert APDS, Rodrigues PF, Albuquerque TM, et al. Vínculo entre enfermeiros e mães de crianças menores de dois anos: percepção de enfermeiros. Ciênc. Saúde Colet. [internet]. 2016 [acesso em 2018 mar 3]; 21(8):2375-2382. Disponível em: https://www.scielosp.org/pdf/cartão/2016.v2ln8/2375-2382. 
18. Baratieri T, Soares LG, Botti ML, et al. Consulta de enfermagem em puericultura: um enfoque nos registros de atendimentos. Rev. Enferm. UFSM [internet]. 2014 [acesso em 2018 jun 14]; 4(1):206-216. Disponível em: https://periodicos.ufsm.br/reufsm/article/ view/8553/pdf.

19. Carvalho EB, Sarinho SW. A consulta de enfermagem no acompanhamento do crescimento e desenvolvimento de crianças na Estratégia Saúde da Família. Rev. Enferm. UFPE [internet]. 2016 [acesso em 2017 dez 12]; 10(6):4804-4812. Disponível em: https://periodicos.ufpe.br/revistas/revistaenfermagem/article/ view/11259.

20. Castro JM, Borja-Cabrera GP. Avaliação da Cobertura Vacinal em Menores de Cinco em um Município do Leste de Minas Gerais. Ensaios e Ciênc.: C. Biológicas, Agrárias e da Saúde. 2017; 21(1):36-41.

21. Prefeitura do Município de Cascavel, Secretaria Municipal e Saúde. Vigilância Epidemiológica. Dados Epidemiológicos. Cascavel: Sesau; 2018.

22. Romão P, Durão F, Valente S, et al. Aleitamento materno: o que mudou em 12 anos. Nascer e Crescer [internet]. 2017 [acesso em 2018 set 18]; 26(3):171-177. Disponível em: http://www.scielo.mec.pt/pdf/nas/ v26n3/v26n3a03.pdf.

23. Pacífico L, Salustiano Q. Fatores associados à duração do aleitamento materno em crianças menores de seis meses. Rev. Bras. Ginecol. Obstet. [internet]. 2012 [acesso em 2018 jul 20]; 34(1): 28-33. Disponível em: http://www.scielo.br/pdf/rbgo/v34nl/a06v34nl.

24. Campagnolo PDB, Louzada MLC, Silveira EL, et al. Práticas alimentares no primeiro ano de vida e fatores associados em amostra representativa da cidade de Porto Alegre, Rio Grande do Sul. Rev. Nutr. [internet]. 2012 [acesso em 2017 nov 22]; 25(4):431-439. Disponível em: http://www.scielo.br/scielo.php?script=sci arttext\&pid=S1415-52732012000400001\&lng=pt\&tl ng=pt.

25. Souza SNDG, Migoto M T, Rossetto EG, et al. Prevalência de aleitamento materno e fatores associados no município de Londrina-PR. Acta Paul. Enferm. [internet]. 2012 [acesso em 2018 jul 19]; 25(1):29-35. Disponível em: http://www.producao.usp.br/handle/BDPI/39116.

26. Pivetta HMF, Braz MM, Pozzebon NM, et al. Prevalência de aleitamento materno e fatores associados: uma revisão de literatura. Rev. Ciênc. Méd. Biol. [internet] 2018 [acesso em 2018 nov 22]; 17(1):95-101. Disponível em: https://portalseer.ufba.br/index.php/ cmbio/article/view/12783/16432.

\footnotetext{
Recebido em 15/03/2019

Aprovado em 04/12/2019

Conflito de interesses: inexistente

Suporte financeiro: Conselho Nacional de Desenvolvimento

Científico e Tecnológico (CNPq)
} 